\title{
ON-THE-FLY INTERPOLATION OF CONTINUOUS TEMPERATURE- DEPENDENT THERMAL NEUTRON SCATTERING DATA IN RMC CODE
}

\author{
Lei ZHENG ${ }^{1, *}$, Zhiyuan FENG ${ }^{1}$, Kan WANG ${ }^{1}$ \\ ${ }^{1}$ Department of Engineering Physics, Tsinghua University \\ 1st Qinghuayuan, Haidian District,Beijing, China \\ z117@mails.tsinghua.edu.cn, \\ fengzy17@mails.tsinghua.edu.cn, wangkan@mail.tsinghua.edu.cn
}

\begin{abstract}
Thermal neutron scattering data have an important influence on the high-fidelity neutronics calculation of thermal reactors. Due to the limited storage capabilities of computers, a discrete ACE representation of the secondary neutron energy and angular distribution has been used for Monte Carlo calculation since the early 1980s. The use of this discrete representation does not produce noticeable effects in the integral calculations such as keff eigenvalues, but can produce noticeable deficiencies for differential calculations. A new continuous representation of the thermal neutron scattering data was created in 2006, but was not widely known. Recently, the continuous representation of the thermal neutron scattering ACE data based on ENDF/B-VIII.0 library was officially released and was available for all users. The new representation shows great difference compared with the discrete one. In order to utilize the more physical and rigorous representation data for high fidelity neutronic-thermohydraulic coupling calculation, the on-the-fly treatment capability was proposed and implemented in RMC code. The two-dimensional linear-linear interpolation method was used to calculate the inelastic scattering cross sections and the secondary neutron energies and angles. The on-the-fly treatment capability was tested by a pressurized water reactor assembly. Results show that the on-the-fly treatment capability has high accuracy, and can be used to consider the temperature feedback in the neutronic-thermohydraulic coupling calculations. However, the efficiency of the on-the-fly treatment still need to be improved in the near future.
\end{abstract}

KEYWORDS: interpolation, continuous thermal neutron scattering data, on-the-fly, RMC

\section{INTRODUCTION}

Thermal neutron scattering data have an important influence on the high-fidelity neutronics calculation of thermal reactors. Since the energy of the thermal neutrons is very low (generally lower than $10 \mathrm{eV}$ ), neutron scattering processes are easily affected by the chemical binding of the target to other nuclides in a material or crystalline structure effects in the interaction with target materials. Meanwhile, neutron scattering events can occur resulting in a gain or loss in energy during the scattering process. All these effects complicate the description and processing of thermal scattering information. Therefore, large amount of computer memory is need to store all the detailed thermal neutron scattering data. A discrete ACE representation of the secondary neutron spectrum and angular distribution processed by NJOY [1]

\footnotetext{
* Corresponding author
} 
has been used for Monte Carlo calculation since the early 1980s due to the limited storage capabilities of computers [2]. The use of the discrete representation does not produce noticeable effects in integral calculations such as keff eigenvalues [3], but can produce unphysical phenomena [2] and noticeable deficiencies for differential calculations [4].

To overcome the inherent deficiencies of the discrete representation, a new continuous ACE representation for the thermal neutron scattering data was firstly created by Los Alamos National Laboratory in 2007 [5]. Continuous representation thermal neutron scattering data based on ENDF/BVII.0 [6] and ENDF/B-VII.1 [7] data libraries were released and available online whereafter, but were not widely known. In the lasted ENDF/B-VIII.0 [8] data libraries, only the continuous ACE representation of the thermal neutron scattering data were provided by Los Alamos National Laboratory (LANL).

In order to utilize the more physical and rigorous representation thermal neutron scattering data for high fidelity neutronics calculations, the continuous thermal neutron scattering data processing capability in Reactor Monte Carlo code RMC [9] has been developed and was introduced in our previous work[10]. In this paper, the on-the-fly treatment capability for the continuous representation thermal neutron scattering data was developed to consider the temperature feedback effect in the neutronic-thermohydraulic coupling calculations. The second part of this paper gives a brief introduction to the continuous thermal neutron scattering data, the third part presents the detailed interpolation method for the on-the-fly treatment capability, the test cases and results are given in the fourth part, and a conclusion was drawn in the final part.

\section{CONTINUOUS THERMAL NEUTRON SCATTERING DATA}

Currently, the continuous ACE representation was designed for both the elastic and the inelastic scattering. In order to generate the continuous representation thermal neutron scattering data, the aceth module in NJOY program has been modified to convert the secondary energy distributions from thermr into probability density functions (PDFs) and cumulative density functions (CDFs) form, PDFs and CDFs are packed into the big inelastic array of the ACE format thermal scattering data. If the users want to obtain the continuous representation ACE data, the weighting option (iwt flag) in card 9 of the ACER module in the NJOY input file should be set to 2 (tabulated). The memory consumption for the continuous ACE representation thermal neutron scattering data is about 10 times larger than the traditional discrete one.

The continuous representation for the inelastic scattering data contains a table of incident energies, a table of inelastic cross sections, a table of pointers to the start of each distribution, a table of the number of points in each distribution, and the secondary energy and angle distributions. Two extra data blocks as well as PDFs/CDFs for the secondary energy distributions are added to the continuous representation compared to the traditional discrete one. A detailed introduction to the format of the continuous representation for the inelastic thermal scattering data can refer to our previous work [10].

\section{ON-THE-FLY TREATMENT CAPABILITY}

Since the inelastic scattering exists in all the thermal materials and plays a key role in the processing of thermal scattering, the present on-the-fly treatment capability mainly focus on the inelastic scattering. The linear-linear interpolation strategy was adopted to calculate the scattering cross sections and the secondary energies and angles. It should be emphasized that the number of grids for the incident energy and secondary energy changes differently with the increase of temperature, the corresponding processing methods were therefore developed for the inelastic scattering cross sections and the secondary energies in this paper. 


\subsection{On-the-fly treatment of cross sections}

For the continuous representation inelastic scattering data, the number of the incident energy grids remains unchanged with the increase of temperature. Therefore, the general two-dimensional linear-linear interpolation method was adopted to calculate the cross sections at target temperature and target energy as shown in Fig 1. $E_{1}$ and $E_{2}$ are the incident neutron energy grids correspond to the target energy $E_{t} . T_{1}$ and $T_{2}$ are the temperature grids correspond to the target temperature $T_{t}$. The inelastic scattering cross sections at target energy $E_{t}$ is calculated at different temperatures firstly, and then the temperature interpolation is performed to calculate the cross section at target temperature $T_{t}$.

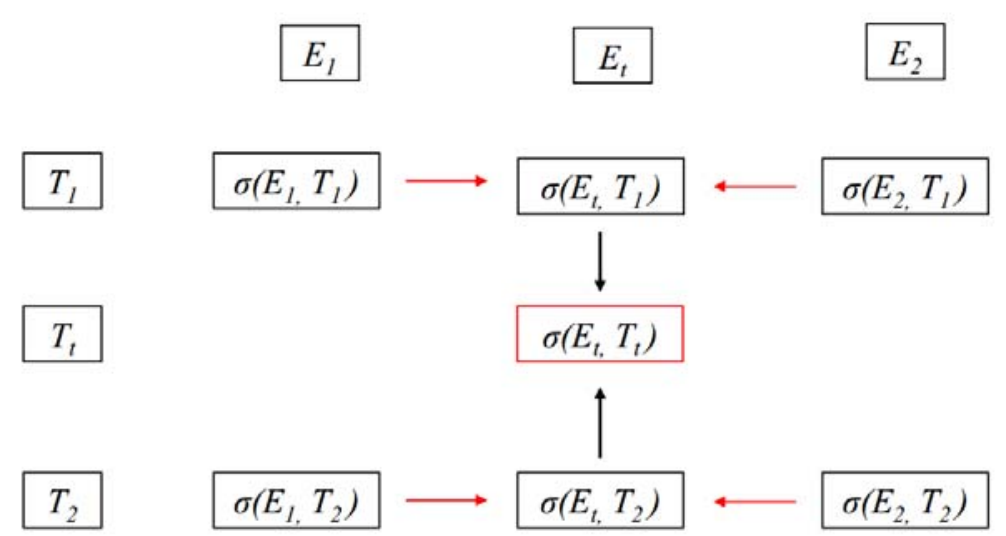

Figure 1. Two-dimensional linear-linear interpolation for the inelastic scattering cross section

\subsection{On-the-fly treatment of secondary energies and angles}

The number of the secondary energy points correspond to incident neutron energies are various from each other at different temperatures for the continuous representation inelastic scattering data. We take light water as an example, the number of the secondary energies correspond to the different incident neutron energies is shown in Fig. 2, and no regular changes are found. 


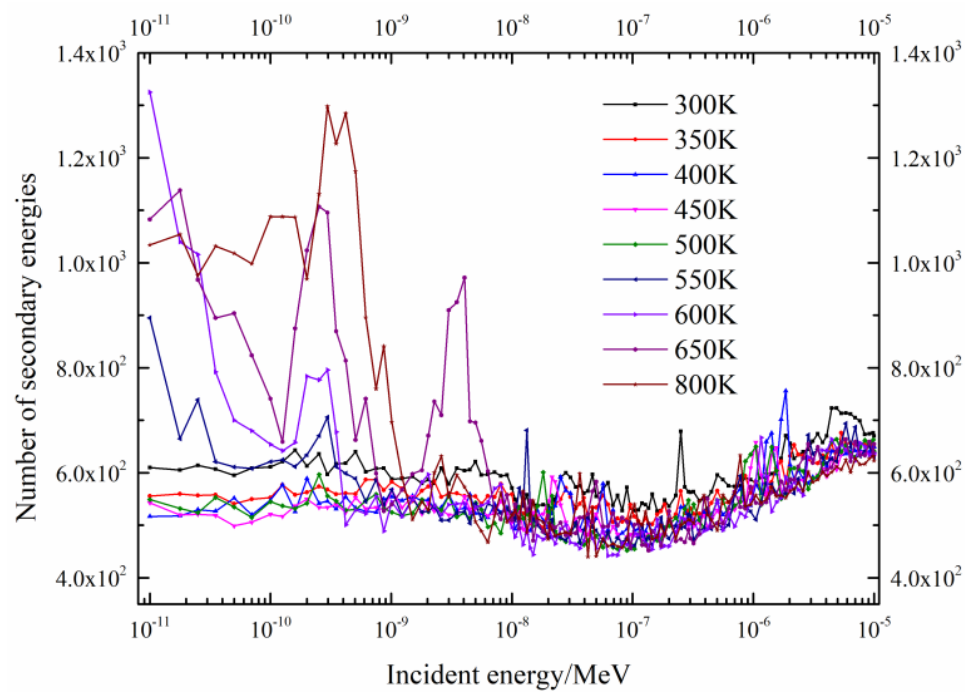

\section{Figure 2. The number of secondary energies corresponding to incident energies for light water inelastic scattering}

Since the secondary energy grids change with the increase of temperature, a treatment method similar to that of the inelastic scattering cross section was adopted. Once the temperature grids for the target temperature is determined, the secondary neutron energy corresponding to the determined incident energy is calculate for the temperature grids using the method introduced in our previous work [10]. The secondary neutron energies and angles are then calculate using the temperature interpolation method. Detailed calculation method is presented as shown below.

First, find the closest temperature grids $T_{1}$ and $T_{2}$ for the interpolation of the target temperature $T_{t}$.

Second, for a given incident neutron energy $E_{\text {in }}$, search the corresponding location of the energy in the energy tables at temperature $T_{1}$ and $T_{2}$, and calculate the energy fraction $r_{i}$, where $\mathrm{i}=1,2$, and correspond to $T_{1}$ and $T_{2} . E_{i, j}$ and $E_{i, j+1}$ are the energy grids in the incident energy table.

$$
\begin{gathered}
E_{i, j}<E_{i n}<E_{i, j+1} \\
E_{i n}=E_{i, j}+r_{i}\left(E_{i, j+1}-E_{i, j}\right)
\end{gathered}
$$

Third, chose the closest energy as the "incident energy", if $r_{i}>0.5, E_{i, j}$ is chosen as the "incident energy", otherwise, $E_{i, j+1}$ is chosen as the "incident energy".

Fourth, sample random number $\xi_{1}$, search the CDFs to determine the outgoing energy grid. $c_{i, l, k}$ and $c_{i, l, k+1}$ are the CDFs corresponding to the "incident energy". It should be emphasized that the same random number must be used for temperature $T_{1}$ and $T_{2}$.

$$
c_{i, l, k}<\xi_{1}<c_{i, l, k+1}
$$


Fifth, calculate the outgoing energy $E_{i}^{\prime}$ and the interpolation fraction $f r_{i}$ for the outgoing angle. $E_{i, l, k}$ and $E_{i, l, k+1}$ are the sampled outgoing energy grids corresponding to $\xi_{1} \cdot p_{i, l, k}$ and $p_{i, l, k+1}$ are the PDFs .

$$
\begin{gathered}
E_{i}^{\prime}=E_{i, l, k}+\frac{\left|\sqrt{p_{i, l, k}^{2}+2\left(\xi_{1}-c_{i, l, k}\right) \frac{p_{i, l, k+1}-p_{i, l, k}}{E_{i, l, k+1}-E_{i, l, k}}}-p_{i, l, k}\right|}{\frac{p_{i, l, k+1}-p_{i, l, k}}{E_{i, l, k+1}-E_{i, l, k}}} \\
f r_{i}=\frac{E_{i}^{\prime}-E_{i, l, k}}{E_{i, l, k+1}-E_{i, l, k}}
\end{gathered}
$$

Sixth, sample the random number $\xi_{2}$, and search the location of the outgoing angle grid. NIL is the inelastic dimensional parameter.

$$
L o c=\operatorname{int}\left[(N I L-1) * \xi_{2}\right]
$$

Seventh, project the outgoing energy up or down, and calculate the final secondary energy. The down projection scheme is given in Eq. (7). The up projection share a similar format to that of the down projection, and the subscript needs to be adjusted to $\mathrm{i}+1$.

$$
E_{i}^{\text {out }}=\left\{\begin{array}{l}
E_{i}^{\prime} \frac{2 E_{i n}-E_{i, j}}{E_{i, j}}, E_{i}^{\prime}>0.5 E_{i, j} \\
E_{i}^{\prime}+E_{i n}-E_{i, j}, E_{i}^{\prime} \leq 0.5 E_{i, j}
\end{array}\right.
$$

Eighth, the linear-linear interpolation method is used to calculate the secondary neutron energy at the target temperature.

$$
E_{t}^{\text {out }}=E_{1}^{\text {out }}+\frac{T_{t}-T_{1}}{T_{2}-T_{1}}\left(E_{2}^{\text {out }}-E_{1}^{\text {out }}\right)
$$

Ninth, sample a random number $\xi_{3}$, and calculate the outgoing angle $\mu_{i}^{\text {out }}, \mu_{i, l, k, L o c}$ is the outgoing angle grid corresponding to the outgoing energy. The detailed calculation of $d$ is complicate and can refer to our previous work [10].

$$
\mu_{i}^{\text {out }}=\mu_{i, l, k, j}+f r_{i} *\left(\mu_{i, l, k+1, L o c}-\mu_{i, l, k, L o c}\right)+d *\left(2 \xi_{3}-1\right)
$$

Finally, the linear-linear interpolation method is used to calculate the secondary angle at the target temperature.

$$
\mu_{t}^{\text {out }}=\mu_{1}^{\text {out }}+\frac{T_{t}-T_{1}}{T_{2}-T_{1}}\left(\mu_{2}^{\text {out }}-\mu_{1}^{\text {out }}\right)
$$




\section{RESULTS AND ANALYSES}

A traditional $17 \times 17$ pressurized water reactor (PWR) assembly is used to test the accuracy and the efficiency of the on-the-fly interpolation strategy, the sketch of the assembly is shown in Fig.3. The assembly has three materials including fuel $\left({ }^{235} \mathrm{U},{ }^{238} \mathrm{U},{ }^{16} \mathrm{O}\right)$, gas gap $\left({ }^{16} \mathrm{O}\right)$ and light water $\left({ }^{1} \mathrm{H},{ }^{16} \mathrm{O}\right)$. The continuous representation thermal neutron scattering data generated by Los Alamos National Laboratory [11] were downloaded and used to consider the thermal scattering effect. And the ENDF/B-VIII.0 based neutron ACE files were generated by using NJOY2016 [12]. The temperature of the light water is set to $500 \mathrm{~K}$, while the fuel and the gas gap are at room temperature.

Four cases adopting the thermal neutron scattering ACE files at different temperatures were performed:

(1) accurate light water $S(\alpha, \beta)$ at $500 K$;

(2) on-the-fly treatment using $\mathrm{S}(\alpha, \beta)$ at both $450 \mathrm{~K}$ and $550 \mathrm{~K}$;

(3) $\mathrm{S}(\alpha, \beta)$ at $450 \mathrm{~K}$;

(4) $\mathrm{S}(\alpha, \beta)$ at $550 \mathrm{~K}$;

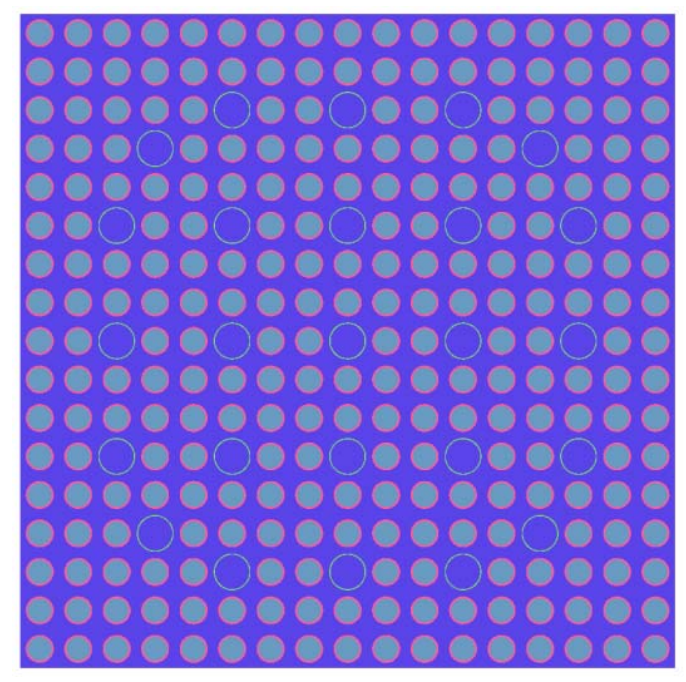

Figure 3. The sketch of the PWR assembly

All calculations were performed using the develop branch of RMC with the on-the-fly treatment for continuous representation thermal scattering data capability implemented. Calculations were performed under the Ubuntu 18.04 system utilizing 6 cores (Intel® Core(TM) i7-7700 CPU @3.60GHz). All cases were run in the criticality mode, using 100 inactive cycles, 400 additional active cycles, and 100000 neutrons per cycle, resulting in the standard deviation lower than $10 \mathrm{pcm}$ for kinf eigenvalues. It should be noted that the calculation time given in Table I is single core calculation time. 
Table I. Comparison of kinf eigenvalues and efficiency

\begin{tabular}{|c|c|c|c|c|}
\hline & kinf & $\mathrm{Std} / \mathrm{pcm}$ & $\Delta \mathrm{k} / \mathrm{pcm}$ & Time/min \\
\hline $\mathrm{S}(\alpha, \beta)$ at $500 \mathrm{~K}$ & 1.415712 & 0.000075 & $/$ & 18.6100 \\
\hline On-the-fly treatment & 1.415631 & 0.000071 & 8.1 & 26.0620 \\
\hline $\mathrm{S}(\alpha, \beta)$ at $450 \mathrm{~K}$ & 1.416268 & 0.000076 & 55.6 & 18.2872 \\
\hline $\mathrm{S}(\alpha, \beta)$ at $550 \mathrm{~K}$ & 1.414673 & 0.000082 & -103.9 & 18.5930 \\
\hline
\end{tabular}

It can be observed from Table I that the kinf value based on the on-the-fly treatment agrees with that based on the accurate light water $\mathrm{S}(\alpha, \beta)$ at $500 \mathrm{~K}$ within 1 sigma. While, the kinf discrepancies are all larger than $50 \mathrm{pcm}$ for $\mathrm{S}(\alpha, \beta)$ at $450 \mathrm{~K}$ and $550 \mathrm{~K}$, which is huge compared with a standard deviation lower than $10 \mathrm{pcm}$. For the time consumption, about $40 \%$ increase is found for the on-the-fly treatment compared with that of the accurate thermal scattering data.
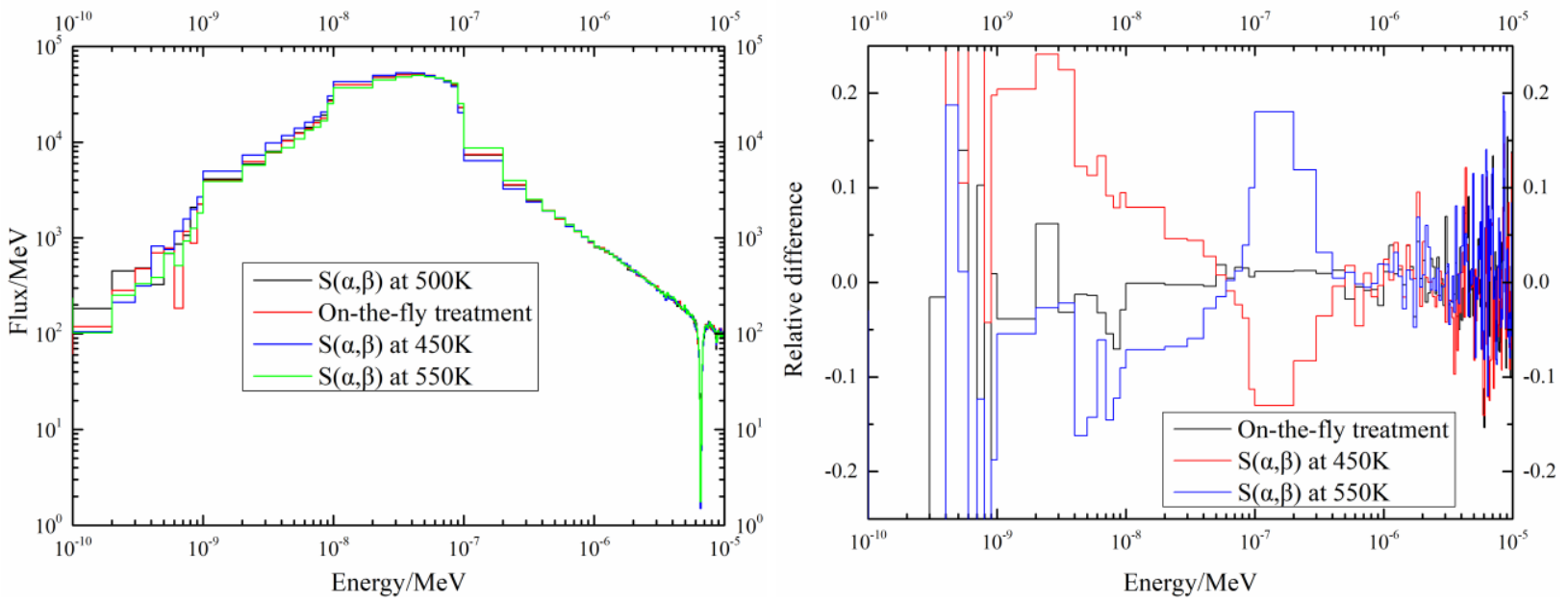

Figure 4. Comparison of energy spectra

Since the maximum energy for thermal treatment is $10 \mathrm{eV}$ for light water, the energy spectra below $10 \mathrm{eV}$ are compared for the four calculation cases in Fig.4. It is found from the spectra that the relative errors between the on-the-fly treatment and the accurate light water $\mathrm{S}(\alpha, \beta)$ at $500 \mathrm{~K}$ are within $5 \%$ for most energy regions, relatively large discrepancies appear at energy regions where the neutron flux are very low and the statistical uncertainties are very large.

The calculation results in Table I and Fig.4 demonstrate that the current on-the-fly treatment for the continuous thermal neutron scattering data capability has high accuracy, and can be used to consider the temperature feedback in the neutronic-thermohydraulic coupling calculations. However, the efficiency of the on-the-fly treatment still needs to be improved in the near future.

\section{CONCLUSIONS}


In this paper, the on-the-fly treatment capability for the continuous representation thermal neutron scattering data has been proposed and implemented in the RMC code. The two-dimensional linear-linear interpolation method was used to calculate the inelastic scattering cross sections and the secondary neutron energies and angles. The on-the-fly treatment capability was tested by a pressurized water reactor assembly. The integral kinf eigenvalues, the efficiency and the energy spectra of the on-the-fly treatment capacity were compared with these of the accurate thermal scattering data at target temperature.

Comparison results show that the on-the-fly treatment capability has high accuracy, and can be used to consider the temperature feedback in the high fidelity neutronic-thermohydraulic coupling calculations. However, the efficiency of the on-the-fly treatment still needs to be improved in the near future. Therefore, our future work mainly focus on the improvement of the efficiency and the implementation of the on-the-fly treatment for the elastic thermal scattering.

\section{ACKNOWLEDGMENTS}

This work is jointly supported by the Science Challenge Project (No. TZ2018001) and the National Natural Science Foundation of China (No. 11775127).

\section{REFERENCES}

1. R.E. Macfarlane, and A.C. Kahler, "Methods for Processing ENDF/B-VII with NJOY," Nucl. Data Sheets, 111(12), pp. 2739-2890 (2010).

2. J.L. Conlin, D.K. Parsons, F.B. Brown, et al, "Continunous $\mathrm{S}(\alpha, \beta)$ Capability in MCNP," Proceedings of 2012 ANS Annual Meeting, Chiago, IL June 24-28, 2012.

3. A.T. Pavlou, F.B. Brown, W.R. Martin, et al, "Comparison of discrete and continuous thermal neutron scattering treatments in MCNP5," Proceedings of the PHYSOR 2012, LaGrange Park, IL, April 15$20,2012$.

4. D.E. Cullen, L.F. Hansen, E.M. Lent, et al, "Thermal Scattering Law Data: Implementation and Testing using the Monte Carlo neutron transport codes COG, MCNP and TART," UCRL-ID-153656, Los Alamos National Laboratory (2003).

5. T.E. Booth, F.B. Brown, J.S. Bull, et al, "MCNP5 1.50 Release Notes," LS-UR-08-2300, Los Alamos National Laboratory (2008).

6. D.K. Parsons and J.L. Conlin, "Release of Continuous Representation for $\mathrm{S}(\alpha, \beta)$ ACE Data," LA-UR12-00800, Los Alamos National Laboratory (2012).

7. D.K. Parsons and J.L. Conlin, "Release of Continuous Representation for $\mathrm{S}(\alpha, \beta)$ ACE Data," LA-UR14-21878, Los Alamos National Laboratory (2014).

8. D.A. Brown, M.B. Chadwick, R. Capote, et al, "ENDF/B-VIII.0: The 8th, Major Release of the Nuclear Reaction Data Library with CIELO-project Cross Sections, New Standards and Thermal Scattering Data," Nucl. Data Sheets, 148, pp.1-148 (2018).

9. Wang K, Li Z, She D, et al, "RMC - A Monte Carlo code for reactor core analysis." Ann. Nucl. Energy, 82, pp. 121-129 (2015).

10. L. Zheng, K. Wang, W. Wang, "Continuous Thermal Neutron Scattering Data Processing Capability in RMC Code". Proceedings of the 3rd Reactor Physics Asia Conference in 2019 (RPHA19), Osaka, Japan, December 2-3, 2019.

11. D.K. Parsons, "NJOY Processing of ENDF/B VIII.0 Thermal Scattering Files," LA-UR-18-25096, Group XCP-5, Los Alamos National Laboratory (2018).

12. R.E. Macfarlane, D.W. Muir, R.M. Boicourt, et al, "The NJOY Nuclear Data Processing System, Version 2016," LA-UR-17-20093, Los Alamos National Laboratory (2017). 